\title{
Evaluating integrated weight linear method to class imbalanced learning in video data
}

\begin{abstract}
With the enormous amount of video data especially with the existence of the noisy and irrelevant information, it would be difficult for a typical detection process to capture a small portion of targeted due to the class imbalance problem. In this paper, class imbalance referred to a very small percentage of positive instance versus negative instances, where the negative instances dominate the detection model, resulting in the degradation of the detection performance. This paper proposed an Integrated Weight Linear (IWL) method that integrate weight linear algorithm (WL) with principle component analysis (PCA) to eliminate imbalanced dataset in soccer video data. PCA is adopted in the first phase with the aim to alleviates the imbalanced data and prepared the reduced instances to the next phase. In the second phase, the reduces instances are refined using the weight linear algorithm. The experiment results using 9 soccer video demonstrate that the integration of PCA and WL is capable to alleviates the imbalanced problem and able to improve classification performance in video data.
\end{abstract}

Keyword: Data mining; Imbalanced data; Video data; Principle component; Weight linear analysis 